\title{
Identification of a novel RPS26 nonsense mutation in a Chinese Diamond-Blackfan Anemia patient
}

\author{
Xiaodong Shi ${ }^{1}$, Xiaolan Huang ${ }^{2}$, Yu Zhang $^{2}$ and Xiaodai Cui ${ }^{2^{*}}$
}

\begin{abstract}
Background: Diamond-Blackfan anemia (DBA), a congenital pure red cell aplasia (PRCA), is characterized by normochromic macrocytic anemia, reticulocytopenia, and nearly absent erythroid progenitors in the bone marrow. DBA10, a subset of DBA, is an autosomal dominant disease caused by a mutation in RPS26. So far, there are 30 disease-causing variants in RPS26 being reported, however, only three of them are small insert mutations.

Case presentation: Here we report a three-month Chinese boy who presents with anemia from postnatal day 2. He was suspected to have Diamond-Blackfan anemia, according to the clinical result. Thus, whole-exome sequencing was performed for precise diagnosis.

Conclusion: Here, a novel insert mutation c.96dupG in RPS26 was identified by whole-exome sequencing, which caused neonatal DBA in a Chinese boy. This is the first case report of a Chinese DBA10 patient who carries a small insertion in the RPS26 gene. These findings expand the mutation diversity of RPS26 and demonstrate the clinical presentations of the Chinese DBA10 patient.
\end{abstract}

Keywords: Diamond-Blackfan, DBA10, RPS26

\section{Background}

Diamond-Blackfan anemia (OMIM:613309) is an inherited pure red cell aplasia (PRCA). It is characterized by normochromic macrocytic anemia, reticulocytopenia, and a near absence of erythroid progenitors in the bone marrow. Most Diamond-Blackfan anemia (DBA) patients have growth retardation. A total of 30 to $50 \%$ of the patients present malformation in craniofacial features, upper limbs, hearts and urinary systems. Symptoms of this disease are heterogenous; they can vary even between affected family members carrying the same mutations [1]. A few genes have been reported to be related to DBA, including RPS19, RPS24, RPS17, RPL35A and RPS26 (MIM 603701). RPS26 encodes a protein that belongs to a protein family called the ribosomal protein family. RPS26 is a crucial component of the $40 \mathrm{~S}$ ribosome-binding site of mRNA [2], a mutation in the

\footnotetext{
* Correspondence: xdcui61@sina.com

${ }^{2}$ Department of Key Laboratory, Capital Institute of Paediatrics, Beijing, China Full list of author information is available at the end of the article
}

RPS26 gene could significantly affect the production of the small subunit of ribosome and cause disease [3].

To date, 30 disease-causing variants in RPS26 have been reported; only three of them are small insertion mutations. Here, we report this experience in the diagnosis of a three-month Chinese DBA patient, according to clinical and genetic test results. This boy presented anemia without any malformations from the postnatal day 2 and had no family history. A novel mutation in the RPS26 gene was identified. To the best of our knowledge, this is the first reported Chinese DBA patient due to a small insertion in the RPS26 gene. Our findings expand the variant diversity of RPS26 and provide more clinical information of Chinese DBA10 patients.

\section{Case presentation \\ Clinical information}

The study was approved by the ethics committee of the Capital Institute of Paediatrics. Written informed consent was obtained from the patient's parents for the publication of this report and any accompanying images. 
The patient was a Chinese boy aged 3 months, who was admitted to the hospital because of anemia. He was born to a non-consanguineous family by normal spontaneous vaginal delivery at full-term with a birth weight of $3.03 \mathrm{~kg}$. He was the only child of the family and no family history of anemia was recorded. This boy presented normochromic anemia with reticulocytopenia and normal platelet count from the postnatal day 2. The patient had no short stature or congenital malformation, and he also presented neither hepatosplenomegal nor lymphadenopathy, had no recurrent infection or bleeding during his stay in the hospital. Further laboratory examinations were performed. The blood routine was: hemoglobin $5.2 \mathrm{~g} / \mathrm{dL}$ (normal range $13.1-17.20 \mathrm{~g} / \mathrm{dL}$ ), the red blood cell $(\mathrm{RBC})$ count $1.62 \times 10^{12} / \mathrm{L}$ (reference range $4.7-6.1 \times 10^{12} / \mathrm{L}$ ) and the reticulocyte $0.43 \%$ (reference 2-6\%); the mean corpuscular volume (MCV), white blood cell and platelet were in the normal range. Bone marrow examination showed a marked erythroid hypoplasia with a paucity of erythroid precursors whose ratio was $1.5 \%$. The granulocyte system and the megakaryocyte system were normal. Testing for the antibody of blood serum, the cytomegalovirus IgM, EB virus and parvovirus B19-DNA tests were negative. No abnormalities was found according to the hemolysis checking. No thymoma was observed regarding to The X-ray and CT scan. The patient had no history of medication or exposure to harmful substances before. According to the clinical information above, he was initially diagnosed with DBA.

\section{Gene sequencing and data analysis}

Gene sequencing was performed by Running Gene Inc. according to the manufacture's protocol. The causal gene was discovered by proband DNA sequencing. DNA was isolated from patient's peripheral blood using DNA Isolation Kit (Bioteke, AU1802). Concentrations of DNA samples were examined by Qubit dsDNA HS Assay Kit (Invitrogen, Q32851) on Qubit fluorometer (Invitrogen, Q33216). Then, genomic DNA were fragmented into 200-300 bp-length pieces by Covaris Acoustic System (Covaris, Massachusetts, USA). Qualified DNA fragments were then processed by following steps to build a DNA library: end-repair, A-tailing and adaptor ligation. After that, DNA libraries were prepared by pre-capture PCR. Probe-captured DNA fragments were then amplified by post-capture PCR. The final products were sequenced on Illumina HiSeq X platform (Illumina, San Diego, USA) as $150 \mathrm{bp}$ paired-end reads, in accordance with its instruction manual.

Data from HiSeq $\mathrm{X}$ were aligned against the human reference genome (hg19) by the BWA Aligner (http:// bio-bwa.sourceforge.net/). The single-nucleotide polymorphisms (SNPs) were examined using GATK software
(Genome Analysis ToolKit) (https://software.broadinstitute.org/gatk/). Candidate variants were annotated using ANNOVAR (annovar.openbioinformatics.org/en/latest/). The potential impacts of single-nucleotide variants (SNVs) were predicted by MutationTaster [4], SIFT [57] and Polyphen-2 [8] programs. According to the ACMG guideline, all mutations were classified to be benign, likely benign, variants of unknown clinical significance (VUS), likely pathogenic and pathogenic.

The candidate causal variants identified via WES were then validated by Sanger sequencing [9]. Co-segregation analyses among the patient's family were also performed. Amplified fragments covering mutated sites were processed using Zymoclean PCR Purification Kit (Zymo Research, USA.) and further sequenced using ABI 3730 DNA Sequencer (SeqGen, CA). Sanger sequencing data were analyzed by Chromas Lite v2.01 (Technelysium Pty Ltd., Tewantin, QLD, Australia).

After gene sequencing and data analysis, a heterozygous variant in RPS26 gene was identified (Additional file 1: Figure S1). This mutation is c.96dupG in chr12:56436301. This frame-shift mutation would cause p.K32fsX5, producing a truncated protein with only 37 amino acids. Sanger sequencing confirmed this mutation as a de novo mutation and demonstrated that neither of the patient's parents is carrier of the mutation. This null variant was interpreted as "pathogenic" according to the variant guidelines of ACMG based upon the following criteria: it is predicted as null variants due to frame-shift (PVS1), this mutation is a de novo variant in a family without disease history (PS2), this mutant is absent in

Table 1 Subtypes of DBA and related genes

\begin{tabular}{ll}
\hline DBA subtypes & Gene/Locus \\
\hline DBA1 & RPS19 \\
DBA2 & 8p23-p22 \\
DBA3 & RPS24 \\
DBA4 & RPS17 \\
DBA5 & RPL35A \\
DBA6 & RPL5 \\
DBA7 & RPL11 \\
DBA8 & RPS7 \\
DBA9 & RPS10 \\
DBA10 & RPS26 \\
DBA11 & RPL26 \\
DBA12 & RPL15 \\
DBA13 & RPS29 \\
DBA14 & TSR2 \\
DBA15 & RPS28 \\
DBA16 & RPL27 \\
DBA17 & RPS27 \\
\hline
\end{tabular}


control (PM2). Therefore, we summarized that this heterozygous null variant in RPS26 is likely contributed to the DBA10 in this patient.

\section{Discussion and conclusions}

Diamond-Blackfan anemia (DBA) is a congenital pure red cell aplasia. Symptoms usually appear within the first year after birth. Normochromic and macrocytic anemia, reticulocytopenia and absent erythroid progenitors in bone marrow are the main characteristics of DBA and are also used as the standards of diagnosis in DBA. Patients with DBA typically present growth retardation and multisystem malformations in $30-50 \%$ of cases [1]. Different subtypes of DBA were reported to be related to various genes. Subtypes of DBA and related genes were listed in Table 1. In several independent studies, RPS26 is responsible for 2.6$6.4 \%$ of DBA patients $[3,10,11]$. Thus, screening the causal gene is essential to achieve precise diagnosis. It also provides a possibility in gene therapy in the future.
RPS26 is a ribosomal protein with 115 amino acids. It is an indispensable component of the $40 \mathrm{~S}$ subunit, which is located close to the mRNA exit site region [12]. RPS26 was demonstrated to regulate the transactivation activity of tumor suppressor p53, which is a transcription factor for genes involved in cell cycle arrest, apoptosis and cellular senescence under stress environments [13]. RPS26 was also reported to be associated with NMD regulator Upf1 in yeast, which indicates a regulatory role of RPS26 in Nonsense-mediated mRNA decay (NMD) process [2]. In addition, RPS26 was documented to be involved in mRNA-specific translation by recognition of the Kozak sequence [13]. The biogenesis of ribosomes might also be affected by RPS26, since a study shows that RPS26 was implicated in the formation of a nucleophosmin [12]. The translation of apoptosis-related protein, biogenesis of ribosomes and mRNA-specific translation may all be implicated in the pathology of DBA10.

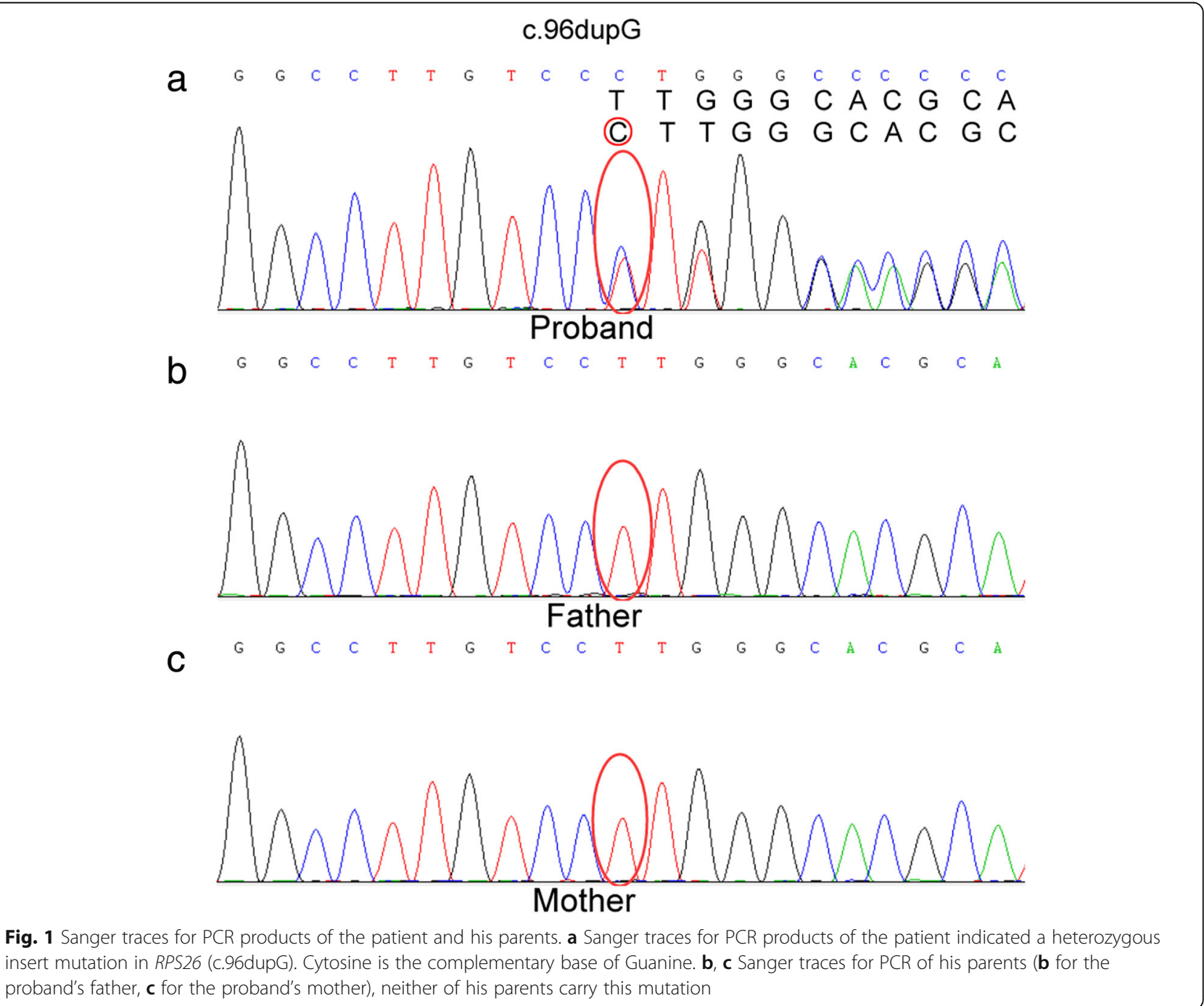


The mutation identified in this study is c.96dupG in the RPS26 gene. This one nucleotide insertion results in a frame-shift mutation p.K32fsX5, producing a protein with only 37 amino acids. This truncated protein is significantly shorter than the wild-type RPS26 protein, which consists of 115 amino acids. DBA10 caused by the RPS26 mutation is an autosomal dominant disease, which means if only one allele carries a disease-causing mutation, this individual would have the disease. Sanger sequencing revealed that neither of the child's parents is a carrier of this mutation (Fig. 1). The mutation detected in the patient is a de novo variant. This also corresponds with the inheritance pattern of the disease.

After searching mutations of RPS26 in HGMD database, 30 variants were found to be recorded. There are 11 point-mutations, 4 splicing mutations, 5 small deletions, 3 small insertions and 7 gross deletions. Then, all the available clinical information of DBA10 patients was summarized. In all DBA10 patients, the onset age varies from 2 weeks to 2 years and 5 months. Most patients presented symptoms of DBA within the first 2 months. Seventeen of 35 patients (48.57\%) presented malformations in the skeleton, heart or urinary system. The patients are not location limited; Czech, Russian, Chinese and Japanese patients were all identified with variants in this gene $[3,14-20]$. The clinical presentation of mutations is also heterogenous. For a prevalently observed mutation c.1A > G (p.M1V), in clinical records of eight patients carrying this mutation, six patients did not present any malformation $[3,16,20]$. One patient was recorded as presenting duplicated pelvocalicon on the right kidney, while another patient was recorded having winged scapula [3], vitamin D deficiency and moderate iron overload [16]. In this report, our patient carried a novel nonsense mutation c.96dupG (p.K32fsX5) and presented severe anemia only 2 days after birth, and was diagnosed as DBA by clinical and genetic diagnosis at 3.5 months. Until then, no significant malformation was observed.

In summary, we identified a novel nonsense mutation in RPS26, c.96dupG (p.K32fsX5) that is associated with autosomal dominant Diamond-Blackfan anemia in a two-day-old neonate. This mutation can result in the production of a truncated protein with 37 amino acids. However, the precise effect of this mutation on protein structure and function remains unknown. Further investigation of mutation effect and the pathology of gene RPS26 on DBA still needs to be carried out.

\section{Additional file}

Additional file 1: Figure S1. Next-Generation Sequencing result of the proband. The BAM file of the NGS sequencing data presented a heterozygous insert mutation of c.96dupG in RPS26 gene. (TIF $7219 \mathrm{~kb}$ )

\section{Abbreviations}

ACMG: American College of Medical Genetics and Genomics; DBA: DiamondBlackfan anemia; MCV: Mean corpuscular volume; NMD: Nonsense-mediated mRNA decay; PRCA: Pure red cell aplasia

\section{Acknowledgements}

We thank Running Gene Inc. for performing Next-generation Sequencing and Sanger Sequencing and providing genetic data. We also thank Xiang Shen from Running Gene Inc. for her critical suggestions and helpful comments during revising. We are also grateful to the patient and the patient's family members for their participation in the study.

\section{Authors' contributions}

CX designed the study. ZW and HX performed the DNA extraction; XS completed the data analysis and other clinical test. XS and CX wrote the manuscript. All authors have read and approved the final manuscript.

\section{Funding}

No funding was obtained for this study.

\section{Availability of data and materials}

We did not use new software, databases, or applications/tools in the manuscript, all results and figures have already provided in the manuscript.

\section{Ethics approval and consent to participate}

All research was approved by the ethics committee of Capital Institute of Paediatrics. The patient's parents have provided written informed consent to undergo genetic testing for this study.

\section{Consent for publication}

The patient's parents gave written consent to studies and publication of clinical information, images and sequencing data.

\section{Competing interests}

The authors declare that they have no competing interests.

\section{Author details}

'Department of Haematology, Capital Institute of Paediatrics, Beijing, China. ${ }^{2}$ Department of Key Laboratory, Capital Institute of Paediatrics, Beijing, China.

Received: 23 January 2019 Accepted: 13 June 2019

Published online: 05 July 2019

\section{References}

1. Landowski M, O'Donohue MF, Buros C, Ghazvinian R, Montel-Lehry N, Vlachos A, et al. Novel deletion of RPL15 identified by array-comparative genomic hybridization in diamond-Blackfan anemia. Hum Genet. 2013; 132(11):1265-74

2. Min EE, Roy B, Amrani N, He F, Jacobson A. Yeast Upf1 CH domain interacts with Rps26 of the $40 S$ ribosomal subunit. RNA (New York, NY). 2013;19(8):1105-15.

3. Doherty L, Sheen MR, Vlachos A, Choesmel V, O'Donohue MF, Clinton C, et al. Ribosomal protein genes RPS10 and RPS26 are commonly mutated in diamond-Blackfan anemia. Am J Hum Genet. 2010;86(2):222-8.

4. Schwarz JM, Cooper DN, Schuelke M, Seelow D. MutationTaster2: mutation prediction for the deep-sequencing age. Nat Methods. 2014;11(4):361-2.

5. Choi $Y$, editor $A$ fast computation of pairwise sequence alignment scores between a protein and a set of single-locus variants of another protein. Proceedings of the ACM Conference on Bioinformatics, Computational Biology and Biomedicine; 2012: ACM.

6. Choi Y, Sims GE, Murphy S, Miller JR, Chan AP. Predicting the functional effect of amino acid substitutions and indels. PLoS One. 2012;7(10):e46688.

7. Choi Y, Chan AP. PROVEAN web server: a tool to predict the functional effect of amino acid substitutions and indels. Bioinformatics. 2015; 31(16):2745-7.

8. Adzhubei IA, Schmidt S, Peshkin L, Ramensky VE, Gerasimova A, Bork P, et al. A method and server for predicting damaging missense mutations. Nat Methods. 2010;7(4):248-9.

9. Sanger F, Nicklen S, Coulson AR. DNA sequencing with chain-terminating inhibitors. Proc Natl Acad Sci U S A. 1977;74(12):5463-7. 
10. Gazda HT, Preti M, Sheen MR, O'Donohue MF, Vlachos A, Davies SM, et al. Frameshift mutation in p53 regulator RPL26 is associated with multiple physical abnormalities and a specific pre-ribosomal RNA processing defect in diamond-blackfan anemia. Hum Mutat. 2012;33(7):1037-44.

11. Wang R, Yoshida K, Toki T, Sawada T, Uechi T, Okuno Y, et al. Loss of function mutations in RPL27 and RPS27 identified by whole-exome sequencing in diamond-Blackfan anaemia. Br J Haematol. 2015;168(6):854-64.

12. Ivanov AV, Gopanenko AV, Malygin AA, Karpova GG. The eS26 protein is involved in the formation of a nucleophosmin binding site on the human 40 ribosomal subunit. Biochim Biophys Acta Proteins Proteom. 2018; 1866(5-6):642-50.

13. Cui D, Li L, Lou H, Sun H, Ngai SM, Shao G, et al. The ribosomal protein S26 regulates p53 activity in response to DNA damage. Oncogene. 2014;33(17):2225-35.

14. Cmejla R, Ludikova B, Sukova M, Blatny J, Pospisilova D. Can mutations in the ribosomal protein S26 (RPS26) gene lead to Klippel-Feil syndrome in diamond-Blackfan anemia patients? An update from the Czech diamondBlackfan Anemia registry. Blood Cells Mol Dis. 2011;46(4):300-1.

15. Farrar JE, Vlachos A, Atsidaftos E, Carlson-Donohoe H, Markello TC, Arceci RJ, et al. Ribosomal protein gene deletions in diamond-Blackfan anemia. Blood. 2011;118(26):6943-51.

16. Gerrard G, Valganon M, Foong HE, Kasperaviciute D, Iskander D, Game L, et al. Target enrichment and high-throughput sequencing of 80 ribosomal protein genes to identify mutations associated with diamond-Blackfan anaemia. Br J Haematol. 2013;162(4):530-6.

17. Gripp KW, Curry C, Olney AH, Sandoval C, Fisher J, Chong JX, et al. Diamond-Blackfan anemia with mandibulofacial dystostosis is heterogeneous, including the novel DBA genes TSR2 and RPS28. Am J Med Genet A. 2014;164a(9):2240-9.

18. Smetanina NS, Mersiyanova IV, Kurnikova MA, Ovsyannikova GS, Hachatryan LA, Bobrynina VO, et al. Clinical and genomic heterogeneity of diamond Blackfan anemia in the Russian Federation. Pediatr Blood Cancer. 2015;62(9):1597-600.

19. Wan Y, Chen X, An W, Ruan M, Zhang J, Chang L, et al. Clinical features, mutations and treatment of 104 patients of diamond-Blackfan anemia in China: a single-center retrospective study. Int J Hematol. 2016;104(4):430-9.

20. Arbiv OA, Cuvelier G, Klaassen RJ, Fernandez CV, Robitaille N, Steele M, et al. Molecular analysis and genotype-phenotype correlation of diamondBlackfan anemia. Clin Genet. 2018:93(2):320-8.

\section{Publisher's Note}

Springer Nature remains neutral with regard to jurisdictional claims in published maps and institutional affiliations.

Ready to submit your research? Choose BMC and benefit from:

- fast, convenient online submission

- thorough peer review by experienced researchers in your field

- rapid publication on acceptance

- support for research data, including large and complex data types

- gold Open Access which fosters wider collaboration and increased citations

- maximum visibility for your research: over $100 \mathrm{M}$ website views per year

At $\mathrm{BMC}$, research is always in progress.

Learn more biomedcentral.com/submissions 